\title{
Topological bifurcations of vortex pair interactions
}

\author{
Nielsen, Anne R.; Andersen, Morten; Hansen, Jesper S.; Brøns, Morten
}

Published in:

Journal of Fluid Mechanics

Link to article, DOI:

10.1017/jfm.2021.191

Publication date:

2021

Document Version

Peer reviewed version

Link back to DTU Orbit

Citation (APA):

Nielsen, A. R., Andersen, M., Hansen, J. S., \& Brøns, M. (2021). Topological bifurcations of vortex pair interactions. Journal of Fluid Mechanics, 917, [2100191]. https://doi.org/10.1017/ffm.2021.191

\section{General rights}

Copyright and moral rights for the publications made accessible in the public portal are retained by the authors and/or other copyright owners and it is a condition of accessing publications that users recognise and abide by the legal requirements associated with these rights.

- Users may download and print one copy of any publication from the public portal for the purpose of private study or research.

- You may not further distribute the material or use it for any profit-making activity or commercial gain

- You may freely distribute the URL identifying the publication in the public portal

If you believe that this document breaches copyright please contact us providing details, and we will remove access to the work immediately and investigate your claim 


\title{
Topological bifurcations of vortex pair interactions
}

\author{
Anne R. Nielsen ${ }^{1} \dagger$, Morten Andersen ${ }^{2}$, Jesper S. Hansen ${ }^{2}$ and \\ Morten Brøns ${ }^{1}$ \\ ${ }^{1}$ Department of Applied Mathematics and Computer Science, Technical University of \\ Denmark, 2800 Lyngby, Denmark \\ ${ }^{3}$ Department of Science and Environment, Roskilde University, 4000 Roskilde, Denmark
}

(Received xx; revised xx; accepted xx)

We investigate vortex pair interactions at low Reynolds numbers. Our analysis is based on the two-dimensional $Q$-criterion and we make use of a topological approach to describe the qualitative changes of the vortex structure. In order to give a complete description of vortex pair interactions we further develop a general bifurcation theory for $Q$-vortices and prove that a threshold for vortex merging may occur when we allow two parameters to vary. To limit the number of free parameters, we study the interactions with two point vortices as initial condition and show that the threshold is a codimension two bifurcation that appears as a cusp singularity on a bifurcation curve. We apply the general theory to the analytically tractable core growth model and conclude that a pair of co-rotating vortices merge only if their strength ratio, $\alpha=\Gamma_{1} / \Gamma_{2}$ is less than 4.58. Below this threshold value, we observe two different regimes in which the merging processes can be described with different sequences of bifurcations. By comparison with Navies-Stokes simulations at different Reynolds numbers, we conclude that the merging threshold varies only slightly for Reynolds numbers up to 100. Furthermore, we observe an excellent agreement between the core growth model and the numerical simulations for Reynolds numbers below 10. We therefore conclude that instead of solving the NavierStokes equation numerically we can, for sufficiently small Reynolds numbers, apply the core growth model as a simple, analytically-tractable model with a low dimension.

\section{Key words: ...}

\section{Introduction}

Studying the fundamental interactions of vortices helps us understand the behaviour of complicated flows which can be encountered in nature. A simple example of an interaction is two-dimensional vortex merging which is a well studied phenomenon in fluid mechanics. For a general review of dynamics and instabilities of vortex pairs, see e.g. Leweke et al. (2016). It is sometimes possible to observe vortex merging visually in experiments and numerical simulations, but it can be difficult to give an accurate mathematical description of the merging process. Early studies of vortex merging mainly focus on merging in inviscid fluids where vortices are defined as vortex patches with constant vorticity (Overman \& Zabusky 1982; Dritschel 1985). The jump of vorticity across vortex boundaries is advected by the velocity field and the problem effectively

$\dagger$ Email address for correspondence: anry@dtu.dk 
becomes one-dimensional. This approach, known as contour dynamics, was originally proposed by Deem \& Zabusky (1978). The conservation of vorticity ensures that the fluid will be divided into regions of uniform vorticity for all time and, in principle, merging is never possible. Dritschel (1986) overcomes this issue by applying contour surgery which is an algorithm allowing two contours enclosing the same uniform vorticity to merge into one if they are close enough together. In this study we will address the problem rigorously in a viscous setting. We identify vortices by the widely used $Q$-criterion (Hunt et al. 1988). In general, a positive $Q$-value implies that the local rotation dominates the strain. In two dimensions $Q$ simplifies to the determinant of the velocity gradient tensor $\nabla \mathbf{u}$ and a vortex is therefore defined as a region where

$$
Q(x, y)=\operatorname{det}(\nabla \mathbf{u}(x, y))>0 .
$$

In this paper, we present a complete topological analysis of merging of Q-vortices in the core growth model. The main result of our analysis is that the merging process can be divided into three different regimes depending on the strength ratio of the vortices. For sufficently high strength ratio, the weakest vortex is supressed by the strong vortex, and no merging as such occurs. For lower strength ratios, there are two different bifurcation sequences leading to merging. The core growth model has previously been used to study vortex merging by Jing et al. (2012) and Andersen et al. (2019). In the following section we review the model and show that it allows to write an analytical expression for $Q$ depending on two parameters, the strength ratio of the two vortices and the time.

We monitor the vortex interactions by looking for bifurcations of the curves bounding the vortices, the level curves $Q(x, y)=0$. Bifurcations occur when singular points appear on these curves. An analysis of all possible perturbations of a given degenerate pattern tells us what we might expect when a given number of parameters are allowed to vary. When only a single parameter is varied, the bifurcations that occur in a robust way are referred to as having codimension one. We have already formulated a complete codimension one theory in earlier studies, see Nielsen et al. (2019) or the brief summary in $\S 3$. Our previous study includes an analysis of a single codimension two phenomenon, but as the core growth model has two free parameters, it is necessary to extend the existing theory with an analysis of another codimension two bifurcation. This further development of the theory can be found in $\S 3.1$. The core growth model has a built-in symmetry that may lead to a special type of codimension two bifurcation; this case is analysed in $\S 3.2$. We compare the results for the core growth model with Navier-Stokes simulations in $\S 4.1$, and find good agreement for low values of the Reynolds number. An analysis of the topological structure of vortex pairs is inextricably linked to the way we choose to define vortices mathematically. There are many definitions of vortices available in the literature, see Zhang et al. (2018) for a review. To our knowledge, this is the first study to analyse vortex pair interactions based on the topology of the $Q$-criterion. Andersen et al. (2019) have previously studied vortex merging from a topological point of view with vortices defined as local extrema of vorticity. This method identifies a vortex by a feature point that does not provide any information about the shape or size of the vortex. Applying the $Q$-criterion might therefore provide some opportunities for a more elaborate analysis. In $\S 5$ we comment on the importance of the vortex criterion.

\section{The core growth model}

We consider an incompressible fluid in an unbounded two dimensional domain governed by the Navier-Stokes equations. In terms of the vorticity, the Navier-Stokes equations can be written as the vorticity transport equation, 


$$
\frac{\partial \omega}{\partial t}=-\mathbf{u} \cdot \nabla \omega+\nu \Delta \omega
$$

where $\nu$ is the kinematic viscosity and $\mathbf{u}$ is the fluid velocity. One of the very few known analytic solutions to (2.1) is the Lamb-Oseen vortex (Saffman 1992),

$$
\omega(r, \theta, t)=\frac{\Gamma}{\pi \sigma(t)^{2}} e^{-r^{2} / \sigma(t)^{2}}, \quad u_{\theta}(r, \theta, t)=\frac{\Gamma}{2 \pi r}\left(1-e^{-r^{2} / \sigma(t)^{2}}\right), \quad u_{r}(r, \theta, t)=0,
$$

with

$$
\sigma(t)=\sqrt{4 \nu t}
$$

The Lamb-Oseen vortex is the solution corresponding to a single point vortex with strength $\Gamma$ as initial condition. The vorticity field $\omega$ is initially concentrated at the origin and diffuses as a Gaussian distribution. For multiple point vortices as initial condition an analytic solution is not available and one would generally refer to numerical simulations of the vorticity transport equation. Instead we investigate the synthetic flow predicted by the core growth model, also known as the multi Gaussian model (Jing et al. 2010; Kim \& Sohn 2012; Andersen et al. 2019). The model assumes that the vorticity of each initial point vortex diffuses symmetrically as an isolated Lamb-Oseen vortex and the centers of the Gaussian vortices move in the velocity field induced by the other diffusing vortices. For two Gaussian vortices, initially centered at $(-d, 0)$ and $(d, 0)$, one can deduce (Kim \& Sohn 2012) that the distance between the centers of the two Gaussian vortices is conserved and the vortices rotate around the a stationary center of vorticity

$$
\left(x_{c v}, y_{c v}\right)=\left(\frac{d\left(\Gamma_{2}-\Gamma_{1}\right)}{\Gamma_{1}+\Gamma_{2}}, 0\right),
$$

with the same time-dependent angular velocity

$$
\frac{d \phi(t)}{d t}=\frac{\Gamma_{1}+\Gamma_{2}}{2 \pi(2 d)^{2}}\left(1-e^{-(2 d)^{2} / \sigma^{2}}\right) .
$$

We notice that the angular velocity tends to zero as $\nu$ or $t$ increases. By integrating $\frac{d \phi(t)}{d t}$ in time we obtain the direction angle as a function of time

$$
\phi(t)=\frac{\Gamma_{1}+\Gamma_{2}}{2 \pi(2 d)^{2} \nu}\left(\frac{\sigma^{2}}{4}-\frac{\sigma^{2}}{4} e^{-(2 d)^{2} / \sigma^{2}}+d^{2} \int_{(2 d)^{2} / \sigma^{2}}^{\infty} \frac{e^{-s}}{s} d s\right) .
$$

We notice that the angular velocity and the direction angle depend on the total of vortex strength $\Gamma_{1}+\Gamma_{2}$ and the distance between the vortices, not on the strength ratio. The positions of the two Gaussian vortex centers $\left(x_{1}(t), y_{1}(t)\right),\left(x_{2}(t), y_{2}(t)\right)$ are given by (2.6), i.e.

$$
\begin{aligned}
& \left(\begin{array}{l}
x_{1}(t) \\
y_{1}(t)
\end{array}\right)=\left(d-x_{c v}\right)\left(\begin{array}{c}
\cos \phi(t) \\
\sin \phi(t)
\end{array}\right)+\left(\begin{array}{c}
x_{c v} \\
0
\end{array}\right), \\
& \left(\begin{array}{l}
x_{2}(t) \\
y_{2}(t)
\end{array}\right)=-\left(d+x_{c v}\right)\left(\begin{array}{c}
\cos \phi(t) \\
\sin \phi(t)
\end{array}\right)+\left(\begin{array}{c}
x_{c v} \\
0
\end{array}\right) .
\end{aligned}
$$

Since the core growth model evolves as a superposition of two Lamb-Oseen vortices, the vorticity field is given as 


$$
\omega(x, y, t)=\frac{\Gamma_{1}}{\pi \sigma^{2}} e^{-d_{1}^{2} / \sigma^{2}}+\frac{\Gamma_{2}}{\pi \sigma^{2}} e^{-d_{2}^{2} / \sigma^{2}},
$$

where

$$
\begin{aligned}
& d_{1}^{2}=\left(x-x_{1}(t)\right)^{2}+\left(y-y_{1}(t)\right)^{2}, \\
& d_{2}^{2}=\left(x-x_{2}(t)\right)^{2}+\left(y-y_{2}(t)\right)^{2} .
\end{aligned}
$$

By solving the Poisson equation $\omega=-\Delta \psi$ we obtain the following streamfunction in the core growth model

$$
\psi(x, y, t)=-\frac{\Gamma_{1}}{4 \pi}\left(\ln \left(d_{1}^{2}\right)+\int_{d_{1}^{2} / \sigma^{2}}^{\infty} \frac{e^{-s}}{s} d s\right)-\frac{\Gamma_{2}}{4 \pi}\left(\ln \left(d_{2}^{2}\right)+\int_{d_{2}^{2} / \sigma^{2}}^{\infty} \frac{e^{-s}}{s} d s\right) .
$$

The core growth model is not an exact solution to the vorticity transport equation in (2.1). However, by inserting the synthetic flow into the equation, we can evaluate the error we make when using the core growth model. From (2.7), (2.8), (2.9) and (2.12) all three terms in the vorticity transport equation can be expressed analytically and by evaluating the limit as $\nu \rightarrow \infty$ we obtain for a fixed $t$ that

$$
\partial_{t} \omega-\nu \Delta \omega+\mathbf{u} \cdot \nabla \omega=\frac{\left(\Gamma_{1}^{2}-\Gamma_{2}^{2}\right) y d}{32 \pi^{2} \nu^{3} t^{3}}+\mathcal{O}\left(\frac{1}{\nu^{4}}\right) \text { as } \nu \rightarrow \infty,
$$

which implies that the core growth model will be accurate for the viscosity going to infinity, i.e. for the Reynolds number going to zero. This will be confirmed by numerical computations in $\S 4.3$. The quality of the approximation will necessarily depend on the value of the fixed $t$. For a smaller $t$ value, a lower Reynolds number is required to achieve the given accuracy. Part of the purpose of this study is to establish an upper limit of the Reynolds number under which it is reasonable to use the core growth model instead of numerically solving the Navier-Stokes equation. It is worth noting that Gallay (2011) proved that on a fixed time interval the solution to the vorticity transport equation, with point vortices as initial conditions, converges uniformly in time to a superposition of Lamb-Oseen vortices as $\nu \rightarrow 0$. Since the core growth model has point vortices as the initial condition, Gallay's result indicates that the model will be relatively accurate also in weakly viscous flow. The model has previously been studied for $R e>>1$, see Jing et al. (2012) for an example.

For simplicity the core growth model will be studied in a co-rotating frame, such that the centers of the Gaussian vortices are fixed at the initial positions. For a given time $t$, the transformation from the co-rotating to the initial frame is determined by a rigid rotation with the angle $\phi(t)$ around the center of vorticity. This guarantees that the topology of the vorticity field, the stream function and hence also the $Q$-field is unchanged when studied in the co-rotating frame. To analyse the core growth model for all possible combinations of vortex strengths and displacements we introduce the following dimensionless variables (denoted with $\sim$ ), $\tilde{x}=x / d, \tilde{y}=y / d, \tilde{\omega}=\omega d^{2} / \Gamma_{2}$, $\tilde{\sigma}=\sigma / d, \tilde{\psi}=\psi d^{2} / \Gamma_{2}$ and $\tilde{\psi}=\psi / \Gamma_{2}$. For simplicity, we are dropping the tildes from now on. In the co-rotating coordinate system the dimensionless vorticity and streamfunction for the core growth model become

$$
\omega(x, y, \alpha, \sigma)=\frac{\alpha}{\pi \sigma^{2}} e^{-\frac{(x+1)^{2}+y^{2}}{\sigma^{2}}}+\frac{1}{\pi \sigma^{2}} e^{-\frac{(x-1)^{2}+y^{2}}{\sigma^{2}}},
$$

and 


$$
\begin{aligned}
\psi(x, y, \alpha, \sigma)= & -\frac{\alpha}{4 \pi}\left(\ln \left((x+1)^{2}+y^{2}\right)+\int_{\frac{(x+1)^{2}+y^{2}}{\sigma^{2}}}^{\infty} \frac{e^{-s}}{s} d s\right) \\
& -\frac{1}{4 \pi}\left(\ln \left((x-1)^{2}+y^{2}\right)+\int_{\frac{(x-1)^{2}+y^{2}}{\sigma^{2}}}^{\infty} \frac{e^{-s}}{s} d s\right)
\end{aligned}
$$

where

$$
\alpha=\frac{\Gamma_{1}}{\Gamma_{2}}, \quad \sigma^{2}=\frac{4 \nu t}{d^{2}}
$$

are the strength ratio of the two vortices and a dimensionless time variable, respectively. As a result the Gaussian vortices are fixed at $(-1,0),(1,0)$. As described in the introduction we will analyse the topological bifurcations of vortex pair interactions by applying the $Q$ criterion. Using (2.15) a closed analytical expression for $Q$ in the co-rotating frame can be directly computed from

$$
Q(x, y, \alpha, \sigma)=\left(\frac{\partial^{2} \psi}{\partial x^{2}}\right)\left(\frac{\partial^{2} \psi}{\partial y^{2}}\right)-\left(\frac{\partial^{2} \psi}{\partial x \partial y}\right)^{2} .
$$

In $\S 4.1$ we will analyse the zero level curves of this function in detail. We notice that the model has a built-in symmetry. The $x$-axis is a line of symmetry in the stream function and hence

$$
Q(x,-y, \alpha, \sigma)=Q(x, y, \alpha, \sigma)
$$

for all values of $x, y, \alpha$ and $\sigma$. Furthermore, it follows from (2.15) that

$$
Q(x, y, \alpha, \sigma)=\alpha Q\left(-x, y, \frac{1}{\alpha}, \sigma\right),
$$

which makes it sufficient to investigate the topological bifurcations of the zero level curves of $Q$ for $|\alpha| \geqslant 1$.

\section{Bifurcation theory for $Q$-vortices}

A general characterization of zero level curves of $Q$ may be applied to any flow situation, regardless of whether it arises from the core growth model or the Navier-Stokes equations. Nielsen et al. (2019) show that there are two types of robust one-parameter bifurcations of the level curves $Q=0$, the authors denoted these as a pinching and a punching bifurcation, see figure 1 . The bifurcations occur when

$$
Q=0, \quad \partial_{x} Q=0, \quad \partial_{y} Q=0,
$$

under the non-degeneracy conditions

$$
\partial_{t} Q \neq 0
$$

and

$$
H^{Q}=\left(\begin{array}{cc}
\partial_{x x} Q & \partial_{x y} Q \\
\partial_{x y} Q & \partial_{y y} Q
\end{array}\right) \text { is non-singular. }
$$

Here $t$ denotes a free parameter. A pinching (punching) bifurcation occurs when $H^{Q}$ is indefinite (definite), and the direction of the bifurcation depends on the sign of $\partial_{t} Q$. A pinching bifurcation is the splitting or merging of two vortices while a punching bifurcation is the creation or disappearance of a single vortex. 
(a)

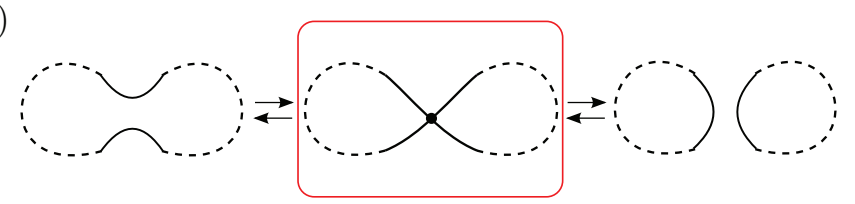

(b)

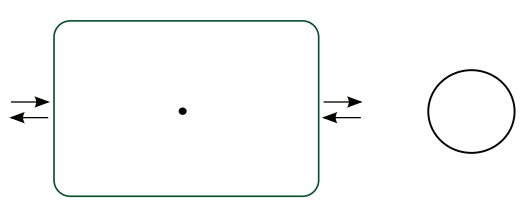

Figure 1: Illustration of the local changes in the structure of the $Q=0$ contour curves during a (a) pinching and a (b) punching bifurcation. The bifurcation states are depicted in red and green boxes. The dashed lines in (a) show an example of a possible global structure during a pinching bifurcation. Note that the empty left panel in (b) illustrates that there are no $Q=0$ contour curves.

In general there is no simple connection between the vorticity $\omega$ and the $Q$-value. If we consider an incompressible fluid at a point $(x, y)$ with $\omega=0$, we notice, however, that

$$
Q=\partial_{x} u \partial_{y} v-\partial_{y} u \partial_{x} v=-\left(\partial_{x} u\right)^{2}-\left(\partial_{y} u\right)^{2} .
$$

Since $Q \leqslant 0$ we conclude that a point with zero vorticity will always be located outside or on the boundary of a $Q$-vortex. By continuity it is therefore impossible to have points with opposite signs of vorticity in the interior of a single vortex. Hence, two vortices can only merge in a pinching bifurcation if they have the same sign of vorticity in the interior.

\subsection{Theoretical description of codimension two bifurcation}

A flow may often depend on several parameters, such as the Reynolds number or a parameter that determines the initial geometry. In this section we consider a flow described as a smooth system depending on two parameters, $t$ and $r$. In this setting the two generic types of one-parameter bifurcations occur when crossing a one dimensional bifurcation curve in the $(t, r)$ parameter space. A codimension two point on one of these bifurcation curves is a point where both parameters are required to take on particular values, so that one of the non-degeneracy conditions in (3.2) or (3.3) is violated. The codimension two bifurcation where $\partial_{t} Q=0$ is analysed in detail in previous studies (Nielsen et al. 2019). In this section we will analyse the other bifurcation phenomenon that occur when $H^{Q}$ is singular with 0 as a simple eigenvalue. For simplicity we choose a coordinate system such that the bifurcation point is located at $(x, y, t, r)=(0,0,0,0)$ and the $H^{Q}$ is a diagonal matrix. We consider a bifurcation point characterized by the following set of degeneracy conditions

$$
Q_{0}=0, \partial_{x} Q_{0}=0, \partial_{y} Q_{0}=0
$$

and

$$
H_{0}^{Q}=\left(\begin{array}{ll}
\partial_{x x} Q_{0} & \partial_{x y} Q_{0} \\
\partial_{x y} Q_{0} & \partial_{y y} Q_{0}
\end{array}\right)=\left(\begin{array}{cc}
0 & 0 \\
0 & \lambda
\end{array}\right),
$$

where subscript 0 is used to denote an evaluation at the bifurcation point $(x, y, t, r)=$ $(0,0,0,0)$ and $\lambda$ is a nonzero parameter. To characterize the structure of the bifurcation curves at the bifurcation point we assume some regularity in the form of the following 
set of non-degeneracy conditions

$$
\partial_{t} Q_{0} \neq 0, \quad \partial_{r} Q_{0} \neq 0, \quad \partial_{x x x} Q_{0} \neq 0,
$$

and that

$$
\left(\begin{array}{cc}
\partial_{x t} Q_{0} & \partial_{x r} Q_{0} \\
\partial_{t} Q_{0} & \partial_{r} Q_{0}
\end{array}\right) \text { is non-singular. }
$$

Based on the above assumptions, we will now analyse the structure of the bifurcation curves in a neighbourhood of the codimension two point. First we consider the following Jacobian

$$
J=\frac{\partial\left(\partial_{y} Q, \partial_{x} Q, Q\right)}{\partial(y, t, r)}=\left(\begin{array}{ccc}
\partial_{y y} Q & \partial_{y t} Q & \partial_{y r} Q \\
\partial_{x y} Q & \partial_{x t} Q & \partial_{x r} Q \\
\partial_{y} Q & \partial_{t} Q & \partial_{r} Q
\end{array}\right)
$$

which simplifies to

$$
J_{0}=\left(\begin{array}{ccc}
\lambda & \partial_{y t} Q_{0} & \partial_{y r} Q_{0} \\
0 & \partial_{x t} Q_{0} & \partial_{x r} Q_{0} \\
0 & \partial_{t} Q_{0} & \partial_{r} Q_{0}
\end{array}\right)
$$

when it is evaluated at the bifurcation point. Since $\lambda \neq 0$ it follows by the non-degeneracy condition (3.7) that $J_{0}$ is non-singular. Hence, we can apply the implicit function theorem to conclude that there exist unique local functions $y=Y(x), t=T(x), r=R(x)$ satisfying

$$
Y(0)=0, \quad T(0)=0, \quad R(0)=0,
$$

and

$$
\begin{aligned}
\partial_{y} Q(x, Y(x), T(x), R(x)) & =0, \\
\partial_{x} Q(x, Y(x), T(x), R(x)) & =0, \\
Q(x, Y(x), T(x), R(x)) & =0 .
\end{aligned}
$$

The functions $T$ and $R$ give a parametric representation of the bifurcation curve in the $(t, r)$ parameter space. The shape of the bifurcation curve is given by the derivatives of $T$ and $R$ at the bifurcation point $x=0$. We now set out to compute these derivatives. By implicit differentiation of the equations in (3.11), we obtain that

$$
J\left(\begin{array}{l}
Y^{\prime}(x) \\
T^{\prime}(x) \\
R^{\prime}(x)
\end{array}\right)=-\left(\begin{array}{c}
\partial_{x y} Q \\
\partial_{x x} Q \\
\partial_{x} Q
\end{array}\right)
$$

which evaluated in $x=0$, gives us

$$
\left(\begin{array}{l}
Y^{\prime}(0) \\
T^{\prime}(0) \\
R^{\prime}(0)
\end{array}\right)=-J_{0}^{-1}\left(\begin{array}{c}
\partial_{x y} Q_{0} \\
\partial_{x x} Q_{0} \\
\partial_{x} Q_{0}
\end{array}\right)=\left(\begin{array}{l}
0 \\
0 \\
0
\end{array}\right)
$$

Since $\left(T^{\prime}(0), R^{\prime}(0)\right)=(0,0)$, we have a non-regular point on the bifurcation curve. To classify the singularity we compute the second order derivatives, which are found by implicit differentiating (3.12) and evaluating the derivatives in $x=0$ :

$$
\left(\begin{array}{c}
Y^{\prime \prime}(0) \\
T^{\prime \prime}(0) \\
R^{\prime \prime}(0)
\end{array}\right)=-J_{0}^{-1}\left(\left(\begin{array}{c}
\partial_{x x y} Q_{0} \\
\partial_{x x x} Q_{0} \\
\partial_{x x} Q_{0}
\end{array}\right)+2 J_{0}^{\prime}\left(\begin{array}{c}
Y^{\prime}(0) \\
T^{\prime}(0) \\
R^{\prime}(0)
\end{array}\right)\right)=-J_{0}^{-1}\left(\begin{array}{c}
\partial_{x x y} Q_{0} \\
\partial_{x x x} Q_{0} \\
0
\end{array}\right)
$$




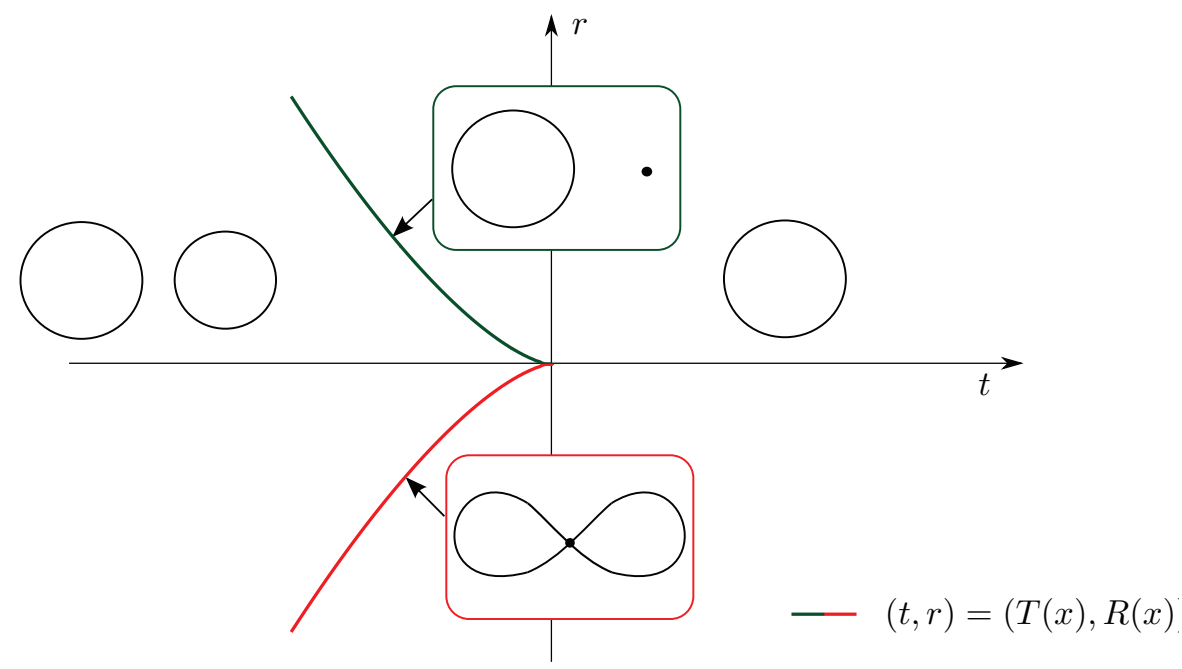

Figure 2: Illustration of a bifurcation curve with a cusp singularity at the codimension two bifurcation point $(t, r)=(0,0)$ satisfying the assumptions in (3.4)-(3.7). The topological structure of the $Q=0$ contour curves are shown in the figure and the bifurcation states are depicted in red and green boxes.

where

$$
J_{0}^{\prime}=\left(\frac{\partial J}{\partial x}+\frac{\partial J}{\partial y} Y^{\prime}(x)+\frac{\partial J}{\partial t} T^{\prime}(x)+\frac{\partial J}{\partial r} R^{\prime}(x)\right)_{0}=\left.\frac{\partial J}{\partial x}\right|_{0} .
$$

Hence, it follows that

$$
\begin{aligned}
T^{\prime \prime}(0) & =-\frac{\partial_{r} Q_{0} \partial_{x x x} Q_{0}}{\partial_{x t} Q_{0} \partial_{r} Q_{0}-\partial_{t} Q_{0} \partial_{x r} Q_{0}}, \\
R^{\prime \prime}(0) & =\frac{\partial_{t} Q_{0} \partial_{x x x} Q_{0}}{\partial_{x t} Q_{0} \partial_{r} Q_{0}-\partial_{t} Q_{0} \partial_{x r} Q_{0}} .
\end{aligned}
$$

From the non-degeneracy conditions (3.6) and (3.7) it is clear that $T^{\prime \prime}(0)$ and $R^{\prime \prime}(0)$ are both well-defined and non-zero and hence we have a quadratic cusp at $(r, t)=(0,0)$ (see e.g. Rutter (2000)). To determine the order $n$ of the cusp we must find the first derivatives of order $n>2$, such that

$$
\frac{T^{(n)}(0)}{R^{(n)}(0)} \neq \frac{T^{\prime \prime}(0)}{R^{\prime \prime}(0)}=-\frac{\partial_{r} Q_{0}}{\partial_{t} Q_{0}} .
$$

We will show that this holds already for $n=3$, which makes the cusp singularity an ordinary cusp. By implicitly differentiating (3.12) again we get that

$$
\left(\begin{array}{l}
Y^{\prime \prime \prime}(0) \\
T^{\prime \prime \prime}(0) \\
R^{\prime \prime \prime}(0)
\end{array}\right)=-J_{0}^{-1}\left(3 J_{0}^{\prime}\left(\begin{array}{c}
Y^{\prime \prime}(0) \\
T^{\prime \prime}(0) \\
R^{\prime \prime}(0)
\end{array}\right)+\left(\begin{array}{c}
\partial_{x x x y} Q_{0} \\
\partial_{x x x x} Q_{0} \\
\partial_{x x x} Q_{0}
\end{array}\right)\right)
$$


Hence, it follows that

$$
\begin{aligned}
T^{\prime \prime \prime}(0) & =\frac{-\partial_{r} Q_{0} A+\partial_{x r} Q_{0} B}{\partial_{x t} Q_{0} \partial_{r} Q_{0}-\partial_{t} Q_{0} \partial_{x r} Q_{0}}, \\
R^{\prime \prime \prime}(0) & =\frac{\partial_{t} Q_{0} A-\partial_{x t} Q_{0} B}{\partial_{x t} Q_{0} \partial_{r} Q_{0}-\partial_{t} Q_{0} \partial_{x r} Q_{0}},
\end{aligned}
$$

where

$$
\begin{aligned}
& A=3 \partial_{x x r} Q_{0} R^{\prime \prime}(0)+3 \partial_{x x t} Q_{0} T^{\prime \prime}(0)+3 \partial_{x x y} Q_{0} Y^{\prime \prime}(0)+\partial_{x x x x} Q_{0}, \\
& B=3 \partial_{x r} Q_{0} R^{\prime \prime}(0)+3 \partial_{x t} Q_{0} T^{\prime \prime}(0)+\partial_{x x x} Q_{0}=-2 \partial_{x x x} Q_{0} .
\end{aligned}
$$

The last non-degeneracy condition in (3.6) then implies that $B$ is non-zero, and hence it follows from (3.7) that at least one of the quantities $T^{\prime \prime \prime}(0)$ and $R^{\prime \prime \prime}(0)$ must be non-zero as well. Assume now that $R^{\prime \prime \prime}(0) \neq 0$ and consider the ratio

$$
\frac{T^{\prime \prime \prime}(0)}{R^{\prime \prime \prime}(0)}=\frac{-\partial_{r} Q_{0} A+\partial_{x r} Q_{0} B}{\partial_{t} Q_{0} A-\partial_{x t} Q_{0} B} .
$$

The following argument is completely identical in the case where $T^{\prime \prime \prime}(0) \neq 0$ and the reciprocal (3.19) is considered. We now assume that

$$
\frac{T^{\prime \prime \prime}(0)}{R^{\prime \prime \prime}(0)}=\frac{T^{\prime \prime}(0)}{R^{\prime \prime}(0)}
$$

However, this implies that

$$
\partial_{x r} Q_{0} \partial_{r} Q_{0} B-\partial_{t} Q_{0} \partial_{x t} Q_{0} B=0,
$$

and since $B \neq 0$ this expression violates the non-degeneracy condition (3.7) and we can conclude that

$$
\frac{T^{\prime \prime \prime}(0)}{R^{\prime \prime \prime}(0)} \neq \frac{T^{\prime \prime}(0)}{R^{\prime \prime}(0)}
$$

This argument concludes the proof that we have an ordinary cusp singularity on a bifurcation curve in the $(t, r)$ parameter space. Since $\partial_{x x x} Q_{0} \neq 0$, it also follows for any $x$ sufficiently close to zero that the Hessian $H^{Q}$ is definite when $x$ has one sign, and indefinite when $x$ has the opposite sign. Hence, the two branches that meet at the cusp singularity are respectively a punching bifurcation curve and a pinching bifurcation curve. A sketch of the bifurcation diagram close to the bifurcation point is shown in figure 2. The orientation of the cusp and the type of bifurcation on each of the two branches will depend on the signs of the non-degenerate quantities in (3.6) and (3.7).

\subsection{Codimension two bifurcation in models with symmetry}

As discussed in $\S 2$ the core growth model has the $x$-axis as a line of symmetry, i.e. $Q(x, y, r, t)=Q(x,-y, r, t)$. This symmetry may lead to a special type of codimension two bifurcation which only occurs in such models. The reason is that the symmetry implies, for any set of non-negative integers $k, l, m$ and $n$, that

$$
\partial_{x}^{k} \partial_{y}^{l} \partial_{t}^{m} \partial_{r}^{n} Q(x, 0, t, r)=0 \quad \text { if } l \text { is an odd number. }
$$

If we consider a bifurcation point satisfying the degeneracy and non-degeneracy conditions described in $\S 3.1$, it would in general not affect the bifurcation phenomenon if we make the analysis in a coordinate system where the $x$ - and $y$-coordinates are interchanged. 


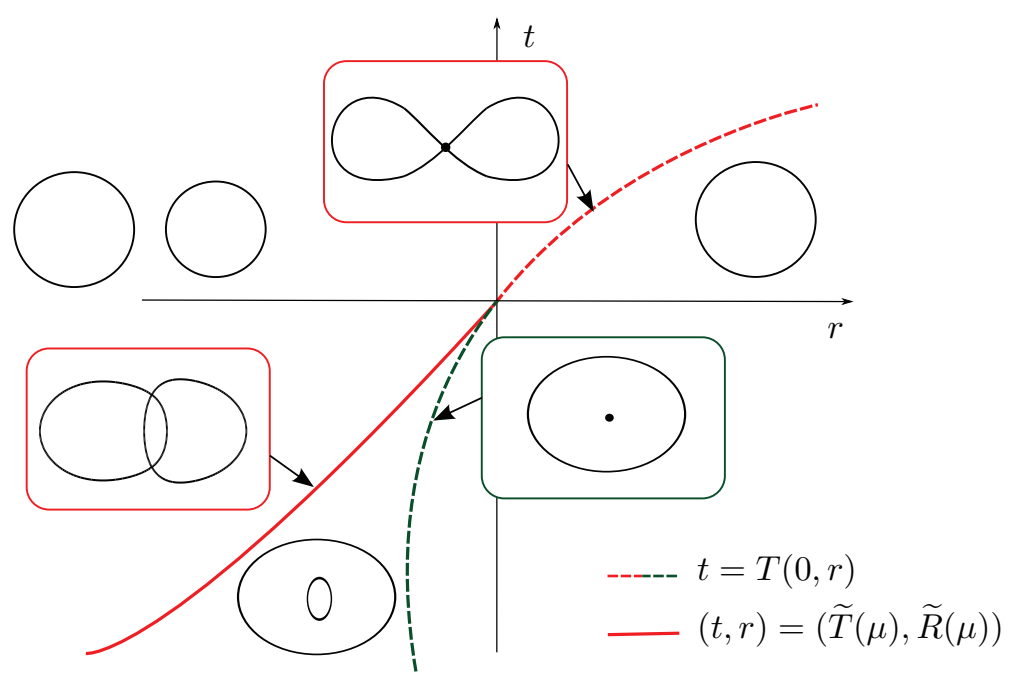

Figure 3: Illustration of a bifurcation diagram in the neighbourhood of a codimension two point satisfying the symmetry condition (3.23). The solid and dashed curves are sketches of the two bifurcation curves meeting with a common tangent line at $(t, r)=(0,0)$. The topological structure of the $Q=0$ contour curves are illustrated in the figure and the bifurcation states are depicted in red and green boxes. See appendix A for further details on $T, \widetilde{T}$ and $\widetilde{R}$.

The symmetry in the core growth model implies, however, that $\partial_{y y y} Q, \partial_{y t} Q$ and $\partial_{y r} Q$ are all zero at any point on the line of symmetry. The non-degeneracy conditions in (3.6) and (3.7) will therefore be violated if the codimension two point is located at the line of symmetry. In Appendix A we analyse this case in detail. The non-degeneracy conditions

$$
\partial_{t} Q_{0} \neq 0, \quad \partial_{r} Q_{0} \neq 0
$$

are kept, and other conditions are imposed to ensure a certain regularity (A 5), (A 6). The analysis in Appendix A shows that two distinct branches of bifurcation curves meet with a common tangent line at the codimension two point and separates the parameter space into three different regions. An example of a bifurcation diagram close to the codimension two point $(t, r)=(0,0)$ is shown in figure 3 . The orientation of the curves and the type of bifurcation on each part of the branches will depend on the signs of the non-degenerate quantities.

\section{Application to vortex pair interactions}

\subsection{Topological bifurcations in the core growth model}

Elsas \& Moriconi (2017) showed that a Gaussian vorticity field has a positive $Q$-value in a circular region with radius $r \approx \sigma / 0.89$. As described in $\S 2$ the core growth model evolves as a superposition of two Gaussian vortices, but since $Q$ does not depend linearly on the flow field bifurcations in the vortex structure can occur. These bifurcations can be tracked by solving the degeneracy conditions (3.1) with $Q$ is given by the analytical expression in (2.17). In the case where $\alpha \geqslant 1$, we obtain the bifurcation diagram shown in figure 4 when the solution is projected onto the $(\sigma, \alpha)$ parameter plane. The bifurcation curves separate the parameter plane into four distinct regions. The vortex structure in each region is illustrated with an example of a $Q=0$ contour curve. The color of a 


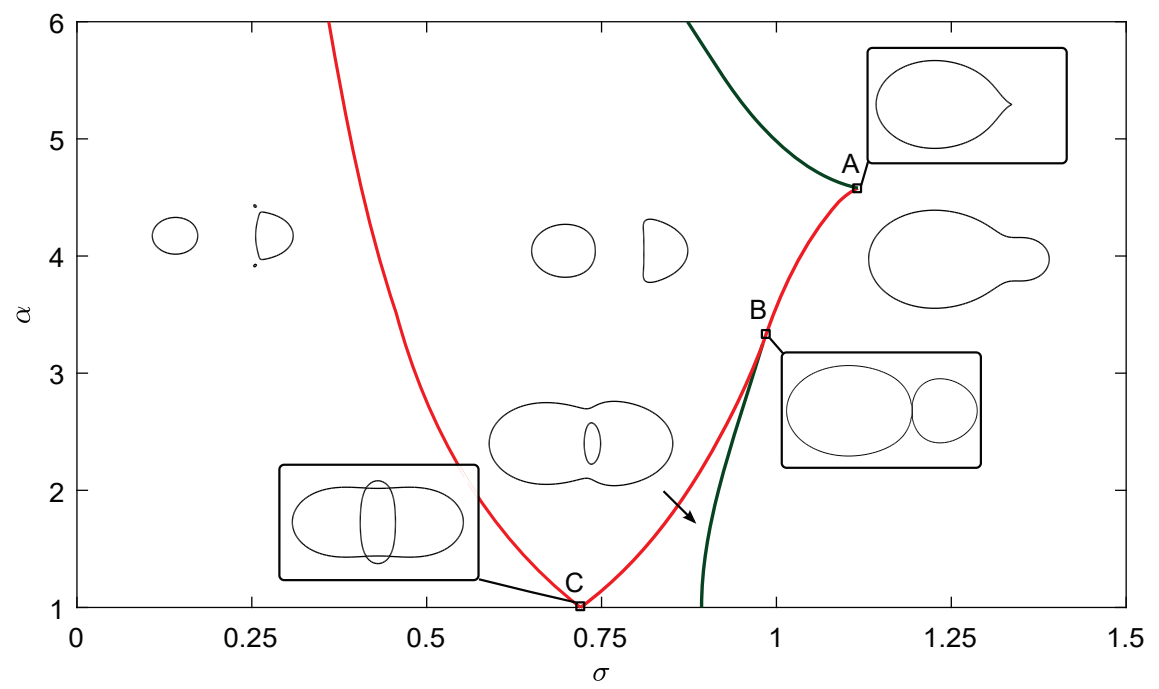

Figure 4: Bifurcation diagram of the merging process in the core growth model for $\alpha \geqslant 1$. The bifurcation curves separate the $(\sigma, \alpha)$ parameter plane into four distinct regions with different vortex topologies. Crossing a green (red) part of the bifurcation curve results in a punching (pinching) bifurcation.

bifurcation curve indicates whether a pinching or a punching bifurcation occur when crossing the curve. The three points $\left(\sigma_{A}, \alpha_{A}\right) \approx(1.12,4.58),\left(\sigma_{B}, \alpha_{B}\right) \approx(0.98,3.37)$, and $\left(\sigma_{C}, \alpha_{C}\right) \approx(0.72,1)$ mark the places where two bifurcation curves collide. These points divide the flow into three different $\alpha$-regimes in which the vortex interactions occur through different robust processes. The temporal evolution of the merging process within each of the three regimes are illustrated by the examples in figure $5 \mathrm{~b}, \mathrm{c}, \mathrm{d}$. The figure also includes the symmetric case where $\alpha=1$. The bifurcation states depicted in the red and green boxes correspond to the vortex structures on the bifurcation curves in figure 4 . The top-down symmetry (2.18) implies that any bifurcations away from the line of symmetry occur simultaneously in pairs. For all values of $\alpha$ the initial and final vortex structure are topologically identical but the temporal evolution is quite different. In the low $\alpha$-regime, $1 \leqslant \alpha<\alpha_{A}$, figure $5 \mathrm{a}$,b, the merging process proceeds in two steps: first a single vortex with a hole is formed by two simultaneous pinching bifurcations. Subsequently the hole disappears in a punching bifurcation. In the intermediate $\alpha$ regime, $\alpha_{B}<\alpha<\alpha_{A}$, figure 5c, the two vortices merge in a single pinching bifurcation. In the high $\alpha$ regime, $\alpha>\alpha_{A}$, figure $5 \mathrm{~d}$, no merging as such occurs, but the weakest vortex is suppressed by the strongest in a punching bifurcation.

When we turn our attention to the common initial vortex structure, we observe two zero level curves of $Q$ located around the Gaussian vortex centers. In addition we notice two smaller vortices that were not immediately expected and grow very slowly in size. For the sake of simplicity, we will only examine them, in the case where $\alpha=1$. Due to the rotational symmetry in this case, they have a fixed location around $(x, y)=(0, \pm 1)$ and the analytical expression of the $Q$-field can easily be evaluated

$$
Q(0, \pm 1,1, \sigma)=\frac{e^{-4 / \sigma^{2}}}{\pi^{2} \sigma^{2}}
$$

It is clear that $(x, y)=(0, \pm 1)$ are singular points of $Q$ in the initial state where $\sigma=0$. Furthermore, $Q( \pm 1,0,1, \sigma)$ has a positive value for any $\sigma>0$ and therefore the small 
(a) $\alpha=1$

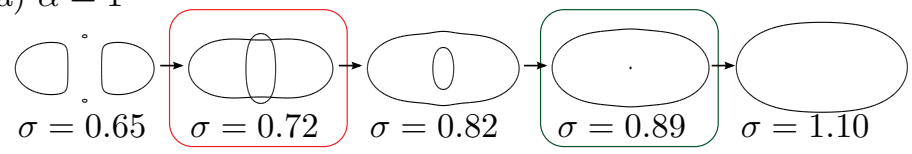

(b) $\alpha=2$

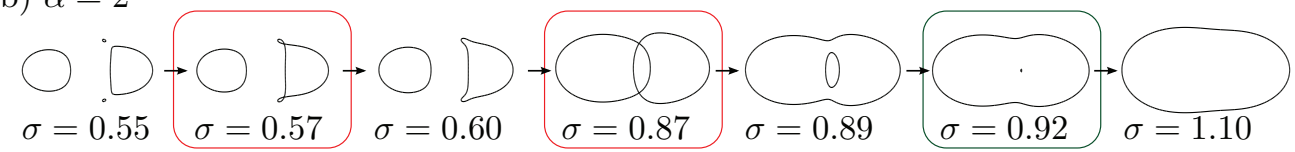

(c) $\alpha=4$

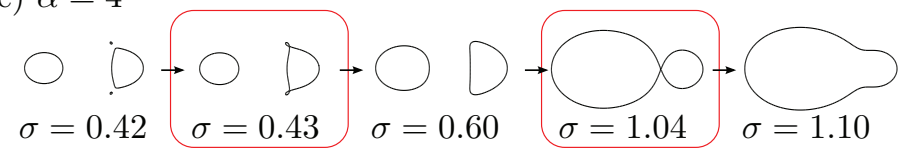

(d) $\alpha=6$

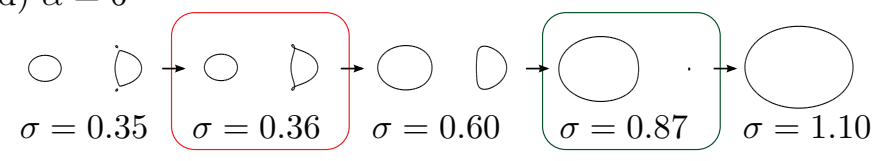

Figure 5: Evolution of the vortex structure at selected values of $\alpha \geqslant 1$. For each value of $\alpha$, all the observed topologies of the $Q=0$ contour curves are shown in the order of evolution. The structurally unstable bifurcation states are depicted in red and green boxes.

vortices are indeed present from the beginning. Furthermore, we see that the value of $Q$ increases slower than any power of $\sigma$. From the examples in figure 5 we see that the small vortices merge with the weakest of the two main vortices in two simultaneous pinching bifurcations that occur when crossing the far left the bifurcation curve in figure 4 .

The key to understanding the complete picture of the vortex pair interactions are the singular points, A, B, C, on the bifurcation curves in figure 4. At these points we observe bifurcations with higher codimension and the corresponding vortex topologies are depicted in black boxes. At $\mathrm{A}$ there is a critical point on the zero level curve of $Q$ at $\left(x_{A}, y_{A}\right) \approx(0.94,0)$. By evaluating $H^{Q}$ precisely at this point, we find that the degeneracy condition (3.5) is satisfied and we can employ our codimension two theory in $\S 3.1$. The two parameters $t$ and $r$ are in this case interpreted as $t=\sigma-\sigma_{A}$ and $r=\alpha-\alpha_{A}$. Based on theory, we conclude that the singular point at $\mathrm{A}$ is an ordinary cusp singularity on the bifurcation curve and the two branches that meet at the cusp singularity are respectively a punching bifurcation curve and a pinching bifurcation curve. This analysis is completely consistent with the result in figure 4 and leads to the same conclusion: a pair of co-rotating vortices merge only if their strength ratio $\alpha=\Gamma_{1} / \Gamma_{2}$ is less than $\alpha_{A}=4.58$. At $\mathrm{B}$ there is a critical point on the zero level curve of $Q$ at $\left(x_{B}, y_{B}\right) \approx(3.91,0)$. Since this critical point is located at the line of symmetry and $H_{B}^{Q}$ satisfy the degeneracy condition (A 2), we can employ our codimension two theory in Appendix A. Based on theory, we conclude that two distinct branches of bifurcation curves meet with a common tangent at the singular point B. Therefore, the point marks the transition between two regimes where merging proceeds as two different sequences of bifurcations exactly as shown in figure 4 . The last singular point at $\mathrm{C}$ is solely due to the rotational symmetry of order 2 when $\alpha=1$ and the point represents a global bifurcation where four distinct bifurcations are restricted to occur simultaneously.

The bifurcation diagram in figure 4 gives us a complete picture of vortex pair inter- 


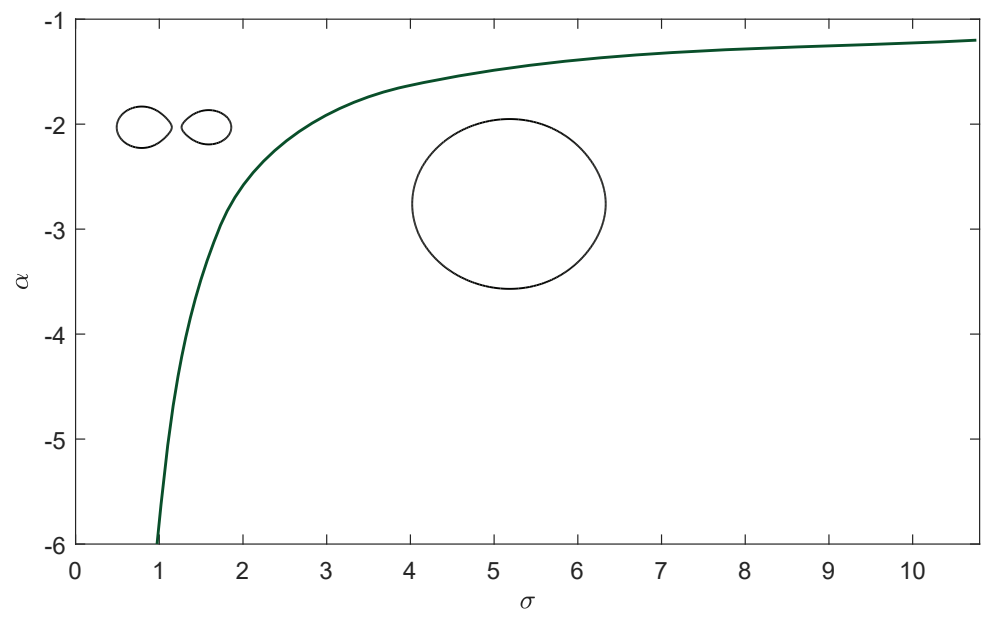

Figure 6: Bifurcation diagram of the merging process in the core growth model for $\alpha<$ -1 . The green bifurcation curve separates the $(\sigma, \alpha)$ parameter plane into two distinct regions with different vortex topologies. The topological structures are illustrated with an example of a $Q=0$ contour curve within each region. On the bifurcation curve one of the vortices disappears in a punching bifurcation.

actions when considering two co-rotating vortices. Since it is only possible to show the diagram for a finite range of $\alpha$, the upper limit of $\alpha=6$ is an arbitrary limit. However, by increasing $\alpha$ significantly, we conclude that the qualitative picture is the same for $\alpha>6$. The limit where $\alpha$ is increased to infinity corresponds to a single Lamb-Oseen vortex, and therefore we expect both bifurcation curves to approach $\sigma=0$ for $\alpha \rightarrow \infty$. For all values of $\alpha$ there is only a single vortex left for $\sigma \geqslant 1.12$. As proven by Gallay \& Wayne (2005) the Lamb-Oseen vortex is an attracting solution for any integrable initial vorticity field. Therefore, we expect that the final vortex region converges to a circular region when $\sigma$ is further increased.

For $\alpha<0$ the vortices have opposite signs of vorticity, and as discussed in $\S 3$ they cannot merge in a pinching bifurcation. This is confirmed by the bifurcation diagram for $\alpha<-1$ in figure 6 . For all values of $\alpha$, the only event is the disappearance of the weakest vortex in a punching bifurcation. When $\alpha$ approaches -1 , the time for the punching bifurcation goes to infinity.

\subsection{Navier-Stokes simulations of vortex pair interactions}

We want to compare the results of the analytical core growth model with NavierStokes simulations subject to the same initial condition. This is done by solving the vorticity transport equation (2.1) numerically. Following Andersen et al. (2019) we do not reparameterize the vorticity transport equation, but control the Reynolds number directly through the kinematic viscosity, $\nu$. In this study we define the Reynolds number as an average of the individual vortex Reynolds numbers

$$
R e=\frac{\left|\Gamma_{1}\right|+\left|\Gamma_{2}\right|}{2 \nu},
$$

which is consistent with earlier studies by Andersen et al. (2019), Meunier et al. (2002) and Jing et al. (2010). Since our system is isolated the total absolute vorticity 


$$
\iint|\omega| d x d y=\left|\Gamma_{1}\right|+\left|\Gamma_{2}\right|
$$

must be conserved. Therefore, we fix $\left|\Gamma_{1}\right|+\left|\Gamma_{2}\right|=10$ in all simulations and control the Reynolds number by varying $\nu$. The conservation of the total absolute vorticity is also monitored as a check of the numerical scheme.

As discussed in $\S 2$ we are primarily interested in comparing the models at low values of Reynolds numbers and we choose to make simulations only for $\mathrm{Re} \leqslant 100$. We restrict the computational domain to the region where $(x, y) \in[-9,9] \times[-9,9]$. Since $\operatorname{Re} \leqslant 100$ and we have a simple square domain a finite difference method with an explicit Euler integrator scheme suffices (Andersen et al. 2019; E \& Liu 1996a,b). If the field variable with index $i j$ is the value at grid point $(i, j)$ we have the iterative scheme

$$
\begin{aligned}
\omega_{i j}^{n+1}\left(\Delta t^{n}\right) & =\omega_{i j}^{n}-\left(\mathbf{u}_{i j}^{n} \cdot\left(\nabla \omega_{i j}^{n}\right)-\nu \nabla^{2} \omega_{i j}^{n}\right) \Delta t^{n}, \\
\nabla^{2} \psi_{i j}^{n+1} & =-\omega_{i j}^{n+1}, \quad u_{i j}^{n+1}=\frac{\partial \psi_{i j}^{n+1}}{\partial y}, \quad v_{i j}^{n+1}=-\frac{\partial \psi_{i j}^{n+1}}{\partial x},
\end{aligned}
$$

where $n$ is the integrator iteration index and $\Delta t^{n}$ the time step used by the scheme at index $n$. We apply an adaptive time step method, where the error estimator is given by the supremum of the absolute differences in the vorticity field using $\Delta t^{n}$ and $\Delta t^{n} / 2$, i.e. err $=\sup \left\{\left|\omega_{i j}^{n}\left(\Delta t^{n}\right)-\omega_{i j}^{n}\left(\Delta t^{n} / 2\right)\right|\right\}$; the relative maximum tolerance is set to $0.1 \%$, and with maximum time step of $10^{-3}$ in simulation time units. The spatial derivatives are approximated by central differences using a $300 \times 300$ grid with grid spacing $\Delta x=0.06$, and we apply periodic boundary conditions. The Poisson problem is solved using the direct method described in Hansen (2011). We note that this simple scheme has been tested against higher order schemes as well as for finite size effects etc. (Andersen et al. 2019).

The initial condition for the core growth model is two Dirac-delta distributions located at $(x, y)=( \pm 1,0)$. Such an initial condition can not be handled by our mesh-based method. Therefore, we consider an initial condition with two slightly diffused Gaussian peaks,

$$
\omega_{i j}^{0}=\frac{\alpha}{\pi \sigma_{0}^{2}} e^{-\frac{\left(x_{i}+1\right)^{2}+y_{j}^{2}}{\sigma_{0}^{2}}}+\frac{1}{\pi \sigma_{0}^{2}} e^{-\frac{\left(x_{i}-1\right)^{2}+y_{j}^{2}}{\sigma_{0}^{2}}},
$$

where $\sigma_{0}=\Delta x$. From the $\omega_{i j}^{0}$ the stream function $\psi_{i j}^{0}$ can be found, which also gives the initial velocity field.

\subsection{Topological bifurcations in Navier-Stokes simulations}

In figure 7 and figure 8 the topological vortex structure is shown for selected simulations with $\operatorname{Re}=10$ and $\operatorname{Re}=100$, respectively. It is important to make clear that the simulations are not performed in a co-rotating frame and we therefore expect the two vortices to rotate relative to each other. In both figures we observe evolution patterns that are qualitatively similar to the ones observed in the core growth model. For $\alpha=1$, the vortex structure still has a rotational symmetry of order two and for increasing values of $\alpha$ we observe three different sequences of topological structures describing the merging process. For the smallest values of $\alpha$, the process involves forming a vortex with a hole in it. For intermediate values of $\alpha$, merging occur as a single pinching bifurcation and no merging is observed for large values of $\alpha$ where the weakest vortex is suppressed in a punching bifurcation. Although there are qualitative similarities, it is clear that 
$\alpha=1$

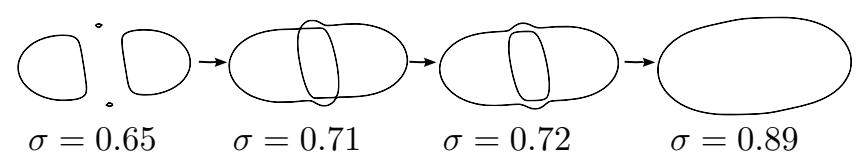

$\alpha=2$

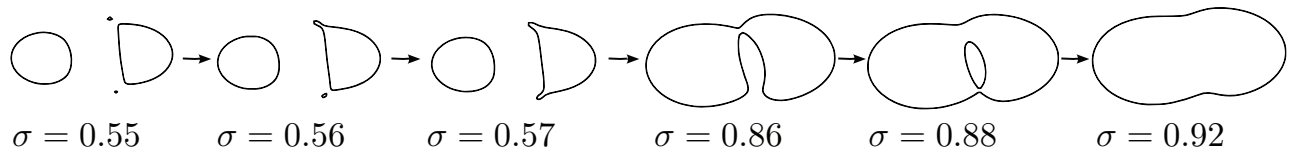

$\alpha=4$

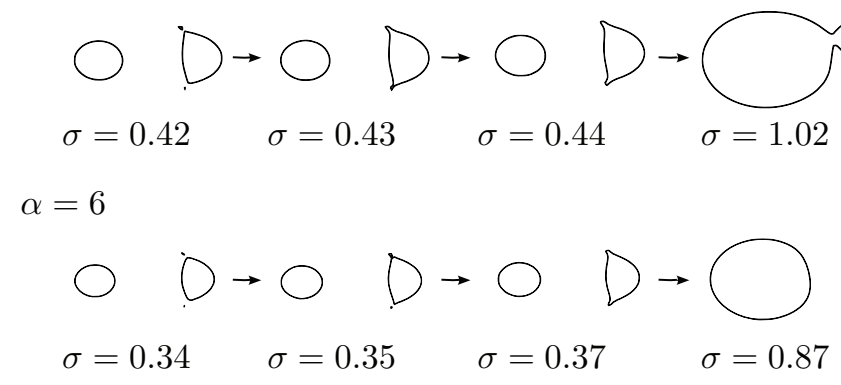

Figure 7: Navier-Stokes simulations at $\mathrm{Re}=10$. The evolution of the vortex structure is shown at selected values of $\alpha \geqslant 1$. For each value of $\alpha$, all the structurally stable topologies of the $Q=0$ contour curve are shown in the order of evolution.

the quantitative picture changes with increasing Reynolds numbers. For both values of Reynolds number there is no line of symmetry in the topological vortex structure. The bifurcations that occur simultaneously in the core growth model are here observed at two distinct values of $\sigma$. We notice that the symmetry is only slightly broken in the case of a low Reynolds number.

For each of the two selected Reynolds numbers, we have performed simulations with more than 30 different values of $1 \leqslant \alpha \leqslant 6$. From each simulation, we have marked the observed bifurcation points in the $(\sigma, \alpha)$ parameter plane and constructed the bifurcation curves shown as the solid lines in figure $9 \mathrm{a}, \mathrm{b}$. The color of the bifurcation curves again indicates the type of bifurcation. For the purpose of comparison the bifurcation curves in the core growth are drawn as dashed lines in the background of the bifurcation diagrams. In both cases, we observe that the codimension two point at B has disappeared. However, this was expected as we do not have a line of symmetry in the Navier-Stokes simulations. With the disappearance of $B$, a new codimension two point $D$ has arisen in both cases. Since a pinching and a punching bifurcation curve meet at D they must form another cusp singularity. The break of symmetry causes the global bifurcation point at $\mathrm{C}$ to separate into two singular points $C_{1}, C_{2}$ where rotational symmetry of order 2 is preserved. The codimension two point at $\mathrm{A}$ is preserved as a cusp singularity but the exact location varies slightly. By recalling that the cusp singularity represents the merging threshold, we conclude that for $\mathrm{Re}=100$ vortex merging is only observed if the strength ratio, $\alpha=\Gamma_{1} / \Gamma_{2}$ is less than $\alpha_{A}=4.05$.

Overall, we observe the same topological structures as seen in the core growth model. Only the bifurcations that were restricted by the built-in symmetry are qualitatively changed. The codimension two points still divide the flow into three different $\alpha$ regimes: 

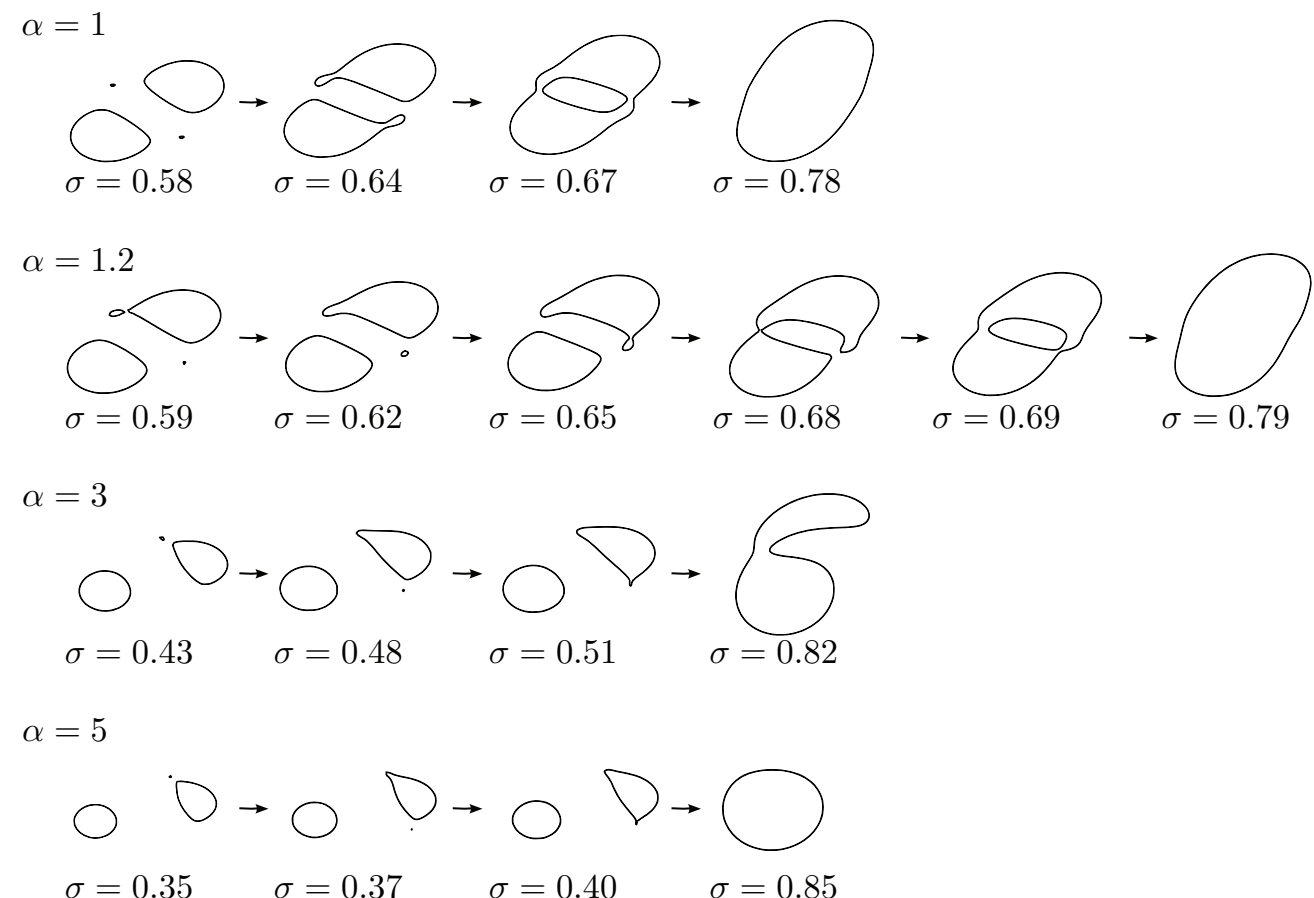

Figure 8: Navier-Stokes simulations at $\mathrm{Re}=100$. The evolution of the vortex structure is shown at selected values of $\alpha \geqslant 1$. For each value of $\alpha$, all the structurally stable topologies of the $Q=0$ contour curve are shown in the order of evolution.

$1 \leqslant \alpha<\alpha_{D}, \alpha_{D} \leqslant \alpha<\alpha_{A}$ and $\alpha>\alpha_{A}$. The examples in figure 7 and 8 are chosen to illustrate the temporal evolution of the merging process within each of the three regimes.

We notice that the small vortices growing around the infinitely degenerate critical points are also present in the Navier-Stokes simulations. Therefore, we conclude that they are not just mathematical artifacts that exist in the core growth model due to a forced symmetry. They, on the other hand, have a significant impact on the observed topological structures. When the small vortices merge with the weaker of the two main vortices, its structure is deformed in a manner that enables the subsequent formation of the interesting vortex structure with a hole inside it. One could argue that the small vortices are artifacts due to the $Q$-criterion. In practice it is common to choose a nonzero threshold to identify the vortex boundaries. The threshold is ideally chosen such that strong vortices are captured while small spurious vortices are ignored. Unfortunately, it is very difficult, if not impossible, to determine a suitable threshold value a priori because the optimal threshold value tends to be problem dependent (see Chen et al. (2015) and Chakraborty et al. (2005)). From the present study it is also clear that the infinitely degenerate critical points out of which the small vortices grow have an effect on the shape of the vortices and we have therefore chosen to stick with the original $Q$-criterion as it is defined in (1.1).

\section{Discussion}

With a topological approach, we revisited the vortex merger problem. The final state of an interacting pair of vortices is known (Gallay \& Wayne 2005) to be a single Lamb- 


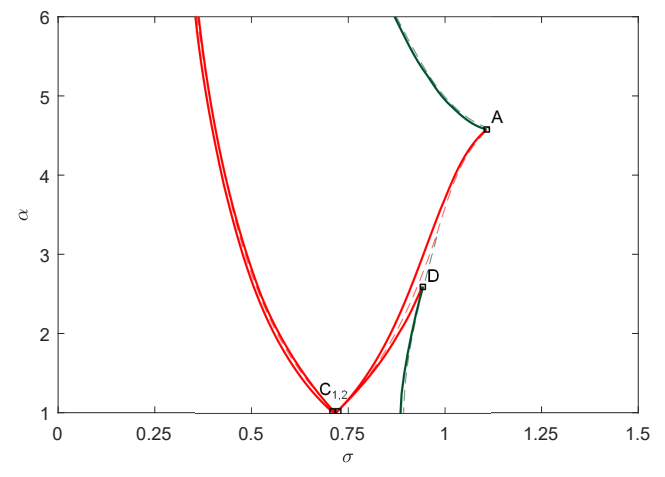

(a) $R e=10$.

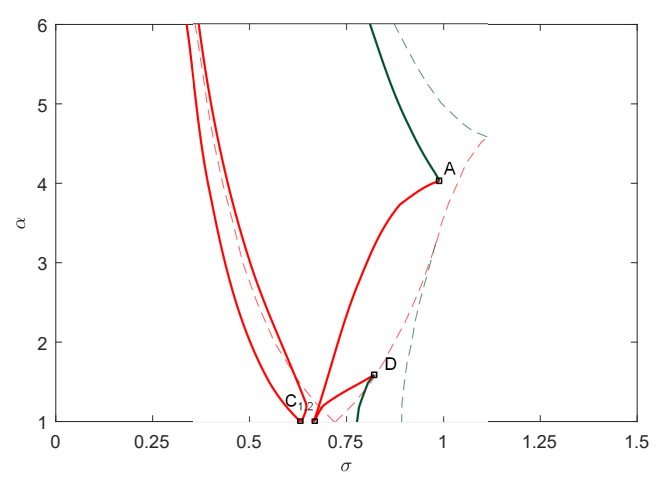

(b) $R e=100$.

Figure 9: Bifurcation diagrams of the merging process in Navier-Stokes simulations with (a) $R e=10$ and (b) $R e=100$. The solid curves are the bifurcation curves in the NavierStokes simulations. The bifurcation curves in the core growth model are drawn as dashed lines for comparison.

Oseen solution. The focus of our studies has therefore been to elucidate the dynamics that take place as the system evolves into the Lamb-Oseen solution. Based on the $Q$ criterion, we have completed a mathematical description that tells us which topological bifurcations we can expect when two parameters are allowed to vary. It has proven to be useful to identify the codimension two points as they organize the bifurcation diagram and divide it into different regimes where different sequences of bifurcations form the merging process. The possible types of bifurcations found from theory also serve as a template that facilitate the construction of a bifurcation diagram for a specific value of Reynolds number. As an example, we know that a pinching and a punching bifurcation curve form a cusp singularity where they meet. Therefore, it is possible to accurately construct the bifurcation curves in figure 9 based on a finite number of Navier-Stokes simulations.

One of the main objectives in this study was to investigate vortex pair interactions using the core growth model. The major advantage of the model is the possibility to determine an analytical expression for $Q$ in the co-rotating frame. With this expression the topology of the $Q=0$ contour curves was easily studied with a precision that made it possible to depict the structurally unstable bifurcation states in figure 5 . The utility of the model was examined by comparing it with Navier-Stokes simulations. Except for the bifurcations that were restricted by the built-in symmetry in the core growth model, we observe the same topological structures in the Navier-Stokes simulations. For Reynolds numbers up to at least 100, the qualitative picture was the same. Furthermore, we observe an excellent quantitative agreement with simulations for Reynolds numbers below 10. Thus, depending on the purpose, there are good opportunities to use core growth model instead of solving the Navier-Stokes equation with low Reynolds numbers.

Most previous studies have focused on symmetric merging of two identical vortices or, to a lesser extent, asymmetric merging with a few examples of different strength ratios, see, among others, Melander et al. (1988), Meunier et al. (2002), Dritschel (1995). In two recent studies by Jalali \& Dritschel $(2018,2020)$ the general inviscid interactions of vortex patches is studied with many examples over a wide parameter space including ratio of sizes and vorticity. Our study is not the first that attempts to describe all interaction scenarios in terms of different flow regimes. Dritschel \& Waugh (1992) identify 
five different flow regimes to characterize the inviscid interaction of two differently sized vortex patches with equal uniform vorticity. These different flow regimes were based on the "efficiency" of the vortex interactions, which was quantified by computing the ratio of the final to initial circulation for each of the vortices. Trieling et al. (2005) show that similar flow regimes can be used to characterize the inviscid interactions of two-dimensional vortices with a continuous vorticity distributions. They notice, however, that the regime boundaries are highly sensitive to the vorticity profile.

It is beyond the scope of this work to study the immediate change in the circulation within the zero level curves of $Q$ during a merging process. A comparison between the initial and long term asymptotic state is, however, possible. We recall that a Gaussian vorticity field has a positive $Q$-value in a circular region with radius $r \approx \sigma / 0.89$ (Elsas \& Moriconi 2017). The circulation within the $Q=0$ level curve of a Gaussian vortex can therefore be directly computed as

$$
\int_{0}^{\sigma / 0.89} \omega(r) 2 \pi r d r=\int_{0}^{\sigma / 0.89} \frac{\Gamma}{\pi \sigma^{2}} e^{-r^{2} / \sigma^{2}} 2 \pi r d r \approx 0.72 \Gamma,
$$

where $\Gamma$ is the total circulation. Thus, the $Q=0$ curve expands at a rate that makes the circulation constant within the $Q$-vortex. For sufficiently small values of $\sigma$ the vorticity field of each of our two initial vortices can be assumed to be Gaussian distributed. Therefore, before any interaction, the circulation of each of the vortices must equal $0.72 \Gamma_{1}$ and $0.72 \Gamma_{2}$, respectively. Since the long time asymptotic state of interacting vortices is a single Gaussian vortex with total circulation $\Gamma_{1}+\Gamma_{2}$, no matter how the transient dynamics evolve, the circulation of the final vortex must equal $0.72\left(\Gamma_{1}+\Gamma_{2}\right)$. Hence, the circulation of the initial vortices is completely transferred to the final surviving vortex. Therefore, merging of viscous $Q$-vortices is always completely efficient, and is complete merging in the sense of Dritschel \& Waugh (1992).

Brandt \& Nomura (2010) use some of the same terms as Dritschel \& Waugh (1992) to describe the flow regimes in a viscous setting at $R e=5000$. All viscous interactions between vortices will eventually result in a single vortex, and therefore only three of the inviscid regimes are considered to occur: complete merger, partial merger and straining out. Brandt \& Nomura (2010) specify three times that are important in the merging process, $t_{c r, 1}, t_{c r, 2}$ and $t_{d e, 2}$. For vortex $i, t_{c r, i}$ indicates the time where the vortex no longer diffuses as a single Gaussian vortex, i.e., the time where the square of the core radius no longer grows linearly. $t_{d e, 2}$ is the time characterizing the destruction of the weaker vortex 2 . The weaker vortex is considered to be destroyed when its core vorticity no longer dominates the imposed strain rate field. This is the case when the $Q$-value is very small at the maximum point of vorticity for the weaker vortex.

Brandt \& Nomura (2010) distinguish between two main regimes, depending on the order of $t_{c r, 2}$ and $t_{d e, 2}$. For large values of $\alpha$, the weak vortex disappears before it gets close to the strong vortex. This is denoted straining-out by Brandt \& Nomura (2010) and characterized by $t_{d e, 2}<t_{c r, 2}$. In our setting, this corresponds to the weakest vortex disappearing in a punching bifurcation. If $t_{c r, 2}<t_{d e, 2}$ the two vortices interact before the weak one disappears. This is denoted merging, and occurs when $\alpha$ is close to 1 . We detect merging in this sense when the two $Q=0$ curves merge in a pinching bifurcation. In both classifications the latter regime is further subdivided. If $t_{c r, 2} \approx t_{c r, 1}$ (complete merger) Brandt \& Nomura (2010) find detrainment of vorticity from both vortices and mutual entrainment of the core into a single vortex. If $t_{c r, 2}<t_{c r, 1}$ (partial merger) there is detrainment from both vortices, but the weaker vortex is destroyed. In our classification, 
there is a regime where a hole is created inside the merged vortex, and one where there is not.

Hence, there are many similarities between the classification by Brandt \& Nomura (2010) and the one we propose on the basis of $Q$ alone, even if the flows are studied at very different Reynolds numbers. In the present study the physics is dominated by diffusion, and convection plays a very small role. In particular, there is no significant filamentation of vorticity (Andersen et al. 2019), and denoting the high $\alpha$ regime where the weak vortex disappears in a punching bifurcation straining-out does not reflect the actual dynamics. However, for higher Reynolds numbers, filamentation will also be detected by the shape of the $Q=0$ contours. A first indication is visible for $\alpha=3$ in figure 8 . The transition to regimes with straining-out and detrainment as Reynolds number is increased can possibly be characterized by further bifurcations in the $Q=0$ contours. From figure 9 we see that the point $\mathrm{D}$ moves to lower values of $\alpha$ as $R e$ is increased. We expect that the regime with a vortex with an inner hole may be very small or disappear completely for higher values of $R e$.

The approach that is common to the studies of Dritschel \& Waugh (1992), Trieling et al. (2005) and Brandt \& Nomura (2010) provides no information about the topological vortex structure during the merging process and it does not define merging as a bifurcation that occurs at a specific time. With our approach, we have the opportunity to connect the process to a rigorous mathematical theory and we avoid a number of choices, such as to define what the core radius is. By using the core growth model we have mapped out all vortex pair interactions with a bifurcation diagram being valid for any choice of vortex strengths with a ratio $|\alpha|=\left|\Gamma_{1} / \Gamma_{2}\right| \leqslant 6$. To our knowledge, a similar bifurcation diagram has only been established once before in a recent study by Andersen et al. (2019). Their results are based on vortices being defined as local extrema of vorticity. Both studies agree that the core growth model matches well with Navier-Stokes simulations for low Reynolds numbers. It is, however, clear that the choice of vortex criterion is crucial to the analysis. While the $Q$-criterion provides information on the physical extent of a vortex, the vorticity criterion only tells us whether or not a feature point for the vortex exists. With the vorticity criterion, it is impossible to distinguish between vortex merging and what we see in this study as suppression of the weakest vortex. Therefore, the threshold for merging and the observed vortex structure with a hole inside are both completely new results that have provided new insights into the intermediate evolution of vortex merging.

Our study has only focused on mapping out all vortex pair interactions with point vortices as initial condition. Inspired by Folz \& Nomura (2017) it would be an obvious continuation of our study to consider two Gaussian vortices with different sizes as initial condition and analyse the effect of differing the vortex area versus the vorticity amplitude. By introducing yet another parameter, the possibility of bifurcations with a higher codimension also arises. It is therefore possible that such a study will require new theoretical considerations.

\section{Acknowledgment}

Financial support from Independent Research Fund Denmark, grant no. 6108-00246B, is gratefully acknowledged.

Declaration of Interests. The authors report no conflict of interest. 


\section{Appendix A. A theoretical description of codimension two phenomena in a symmetric model}

In this appendix we analyse the codimension two bifurcation which is briefly discussed in $\S 3.2$. We consider a flow with symmetry such that $Q(x, y, r, t)=Q(x,-y, r, t)$. For any set of non-negative integers $k, l, m$ and $n$ it then follows that

$$
\partial_{x}^{k} \partial_{y}^{l} \partial_{t}^{m} \partial_{r}^{n} Q(x, 0, t, r)=0 \quad \text { if } l \text { is an odd number. }
$$

The special codimension two bifurcation will only occur on the line of symmetry, $y=0$, since the conditions in (A 1) only applies at the line of symmetry. For simplicity we analyse the phenomenon in a coordinate system where the bifurcation point is located at $(x, y, t, r)=(0,0,0,0)$ and, as before, we use subscript 0 to denote evaluation at the bifurcation point. We consider a bifurcation point that is characterized by the following set of degeneracy conditions

$$
Q_{0}=0, \partial_{x} Q_{0}=0, \partial_{y} Q_{0}=0,
$$

combined with the non-degeneracy condition in (3.3) being violated such that

$$
H_{0}^{Q}=\left(\begin{array}{cc}
\partial_{x x} Q_{0} & \partial_{x y} Q_{0} \\
\partial_{x y} Q_{0} & \partial_{y y} Q_{0}
\end{array}\right)=\left(\begin{array}{cc}
\lambda & 0 \\
0 & 0
\end{array}\right)
$$

for some $\lambda \neq 0$. In this section we prove that if the bifurcation point also satisfies the following non-degeneracy conditions

$$
\begin{gathered}
\partial_{t} Q_{0} \neq 0, \partial_{r} Q_{0} \neq 0 \\
\partial_{x y y} Q_{0} \frac{\partial_{x t} Q_{0} \partial_{r} Q_{0}-\partial_{x r} Q_{0} \partial_{t} Q_{0}}{\partial_{x x} Q_{0} \partial_{t} Q_{0}}-\partial_{y y t} Q_{0} \frac{\partial_{r} Q_{0}}{\partial_{t} Q_{0}}+\partial_{y y r} Q_{0} \neq 0
\end{gathered}
$$

and

$$
-3 \frac{\left(\partial_{x y y} Q_{0}\right)^{2}}{\partial_{x x} Q_{0}}+\partial_{y y y y} Q_{0} \neq 0,
$$

then two distinct branches of bifurcation curves meet tangentially at $(t, r)=(0,0)$, as illustrated in figure 3 .

We begin the proof by considering the following Jacobian

$$
J=\frac{\partial\left(\partial_{x} Q, Q\right)}{\partial(x, t)}=\left(\begin{array}{cc}
\partial_{x x} Q & \partial_{x t} Q \\
\partial_{x} Q & \partial_{t} Q
\end{array}\right),
$$

which simplifies to

$$
J_{0}=\left(\begin{array}{cc}
\lambda & \partial_{x t} Q_{0} \\
0 & \partial_{t} Q_{0}
\end{array}\right)
$$

when it is evaluated at the bifurcation point. Since $\lambda \neq 0$ it follows from the nondegeneracy condition (A 4 ) that $J_{0}$ is non-singular. Hence, we can apply the implicit function theorem to conclude that there exist unique local functions $x=X(y, r), t=$ $T(y, r)$ satisfying

$$
X(0,0)=0, \quad T(0,0)=0
$$

and

$$
\partial_{x} Q(X(y, r), y, T(y, r), r)=0, \quad Q(X(y, r), y, T(y, r), r)=0 .
$$

Since $Q$, and hence also $\partial_{x} Q$, are symmetric functions in $y$, it follows that 


$$
\begin{gathered}
\partial_{x} Q(X(-y, r),-y, T(-y, r), r)=\partial_{x} Q(X(-y, r), y, T(-y, r), r)=0, \\
Q(X(-y, r),-y, T(-y, r), r)=Q(X(-y, r), y, T(-y, r), r)=0 .
\end{gathered}
$$

By comparing the expressions in (A 10) and (A 11) and based on the uniqueness of $X$ and $T$ we can conclude that $X$ and $T$ are also symmetric functions in $y$, i.e. $X(-y, r)=X(y, r)$ and $T(-y, r)=T(y, r)$. In order to give a parametric representation of the bifurcation curves we must solve the equation

$$
\partial_{y} Q(X(y, r), y, T(y, r), r)=0
$$

for $y$ in terms of $r$. It follows from (A 1 ) that

$$
\partial_{y} Q(X(0, r), 0, T(0, r), r)=0,
$$

for any $r$. It follows that there exists a branch of bifurcation points which will remain on the line of symmetry $y=0$. The curve, $t=T(0, r)$, gives us a parametric representation of this branch in the $(t, r)$ parameter space. Since $T$ is a continuously differentiable function the slope of the tangent at $r=0$ can be determined by implicit differentiating (A 10), yielding

$$
\partial_{r} T_{0}=-\frac{\partial_{r} Q_{0}}{\partial_{t} Q_{0}}
$$

In figure 3 this branch of bifurcation points is illustrated by the dashed green curve. We expect, however, that there are other solutions of (A 12), where the bifurcation points are leaving the line of symmetry. Since $Q$ is a symmetric function in $y, \partial_{y} Q$ must be an antisymmetric function in $y$. Hence, a Taylor expansion of the left hand side of (A 12) based at $(y, r)=(0,0)$ has the form

$$
\partial_{y} Q(X(y, r), y, T(y, r), r)=A y r+B y^{3}+C y r^{2}+\mathcal{O}(4),
$$

where the terms of order 4 (or higher) contains only odd powers of $y$. The coefficients $A, B$ and $C$ can be expressed solely in terms of derivatives of $Q$ evaluated at the bifurcation point. Only $A$ and $B$ will play a role and are given in (A 16) and (A 17). We assume they are non-zero, and that is exactly the non-degeneracy conditions in (A 5) and (A 6).

$$
A=\partial_{x y y} Q_{0} \frac{\partial_{x t} Q_{0} \partial_{r} Q_{0}-\partial_{x r} Q_{0} \partial_{t} Q_{0}}{\partial_{x x} Q_{0} \partial_{t} Q_{0}}-\partial_{y y t} Q_{0} \frac{\partial_{r} Q_{0}}{\partial_{t} Q_{0}}+\partial_{y y r} Q_{0} \neq 0
$$

and

$$
B=-3 \frac{\left(\partial_{x y y} Q_{0}\right)^{2}}{\partial_{x x} Q_{0}}+\partial_{y y y y} Q_{0} \neq 0
$$

To obtain a parametric representation of the second branch of the bifurcation curve, we put $r=\mu y$ and define

$$
F(y, \mu)=\frac{\partial_{y} Q(X(y, \mu y), y, T(y, \mu y), \mu y)}{y^{2}} .
$$

From this definition we notice that $F(-y,-\mu)=-F(y, \mu)$. Therefore, $F$ has the following Taylor expansion based at $(y, r)=(0,0)$,

$$
F(y, \mu)=A \mu+B y+\mathcal{O}(3) .
$$


Since $F(0,0)=0$ and $\partial_{y} F(0,0)=B \neq 0$, the implicit function theorem establishes the existence of a unique solution $y=Y(\mu)$ satisfying that $Y(0)=0$,

$$
F(Y(\mu), \mu)=0
$$

and hence

$$
F(-Y(-\mu), \mu)=-F(Y(-\mu),-\mu)=0 .
$$

By comparing the expressions in (A 20) and (A 21) and based on the uniqueness of $Y$ we conclude that $Y$ is an odd function. From the definition of $Y$ it is clear that $(y, r)=(Y(\mu), \mu Y(\mu))$ is a solution to the equation in (A 12). A second branch of bifurcation points in the $(t, r)$ parameter space can therefore be determined by the parametric equations

$$
\begin{aligned}
t & =\widetilde{T}(\mu)=T(Y(\mu), \mu Y(\mu)), \\
r & =\widetilde{R}(\mu)=\mu Y(\mu) .
\end{aligned}
$$

It follows from the definitions of $T$ and $Y$ that $\widetilde{T}(0)=0$ and $\widetilde{R}(0)=0$. Furthermore, we see that

$$
\widetilde{T}(-\mu)=T(Y(-\mu),-\mu Y(-\mu))=T(-Y(\mu), \mu Y(\mu))=T(Y(\mu), \mu Y(\mu))=\widetilde{T}(\mu)
$$

and

$$
\widetilde{R}(-\mu)=-\mu Y(-\mu)=\mu Y(\mu)=\widetilde{R}(\mu) .
$$

Since both $\widetilde{T}$ and $\widetilde{R}$ are even functions, it follows that $\widetilde{R}^{\prime}(0)=\widetilde{T}^{\prime}(0)=0$ and it is clear that $(t, r)=(0,0)$ must be a singular point on the curve. The parameter values $\mu>0$ and $\mu<0$ correspond to the two branches on either side of the singular point. Since $\widetilde{T}$ and $\widetilde{R}$ are even functions it is clear that the two branches must coincide and $(t, r)=(0,0)$ is in fact an endpoint of the parametric curve as illustrated in figure 3 . The tangent to the curve is not well-defined at the endpoint but we notice that the limiting tangent direction can be computed as

$$
\lim _{\mu \rightarrow 0}\left(\frac{\widetilde{T}^{\prime}(\mu)}{\widetilde{R}^{\prime}(\mu)}\right)=\frac{\widetilde{T}^{\prime \prime}(0)}{\widetilde{R}^{\prime \prime}(0)}
$$

if $\widetilde{R}^{\prime \prime}(0) \neq 0$. Thus, we must compute the second order derivatives of $\widetilde{T}$ and $\widetilde{R}$ at $\mu=0$. By implicit differentiating (A 20) we obtain

implying that

$$
Y^{\prime}(0)=-\frac{A}{B} \neq 0
$$

$$
\begin{aligned}
& \widetilde{T}^{\prime \prime}(0)=2 Y^{\prime}(0) \partial_{r} T_{0}=-\frac{2 A \partial_{r} Q_{0}}{B \partial_{t} Q_{0}} \neq 0, \\
& \widetilde{R}^{\prime \prime}(0)=2 Y^{\prime}(0)=-\frac{2 A}{B} \neq 0 .
\end{aligned}
$$

Summing up we get that

$$
\lim _{\mu \rightarrow 0}\left(\frac{\widetilde{T}^{\prime}(\mu)}{\widetilde{R}^{\prime}(\mu)}\right)=\frac{\frac{2 A \partial_{r} Q_{0}}{B \partial_{t} Q_{0}}}{-\frac{2 A}{B}}=-\frac{\partial_{r} Q_{0}}{\partial_{t} Q_{0}}
$$


which implies that both branches of the bifurcation curve share a common tangent line in $(t, r)=(0,0)$ as illustrated in figure 3 . The orientation of the bifurcation curves and the type of bifurcation on each part of the branches will depend on the signs of the non-degenerate quantities.

\section{REFERENCES}

Andersen, M., Schreck, C., Hansen, J. S. \& Brøns, M. 2019 Vorticity topology of vortex pair interactions at low reynolds numbers. European Journal of Mechanics, B/fluids 74, $58-67$.

Brandt, Laura K. \& Nomura, Keiko K. 2010 Characterization of the interactions of two unequal co-rotating vortices. Journal of Fluid Mechanics 646, 233-253.

Chakraborty, P., Balachandar, S. \& Adrian, R. J. 2005 On the relationships between local vortex identification schemes. Journal of Fluid Mechanics 535, 189-214.

Chen, Q., Zhong, Q., QI, M. \& Whang, X. 2015 Comparison of vortex identification criteria for planar velocity fields in wall turbulence. Physics of Fluids 27 (8), 085101.

Deem, G.S. \& Zabusky, N.J. 1978 Vortex waves - stationary v states, interactions, recurrence, and breaking. Physical Review Letters 40 (13), 859-862.

Dritschel, D. G. 1985 The stability and energetics of corotating uniform vortices. Journal of Fluid Mechanics 157, 95.

Dritschel, D. G. 1986 Contour surgery: A topological reconnection scheme for extended integrations using contour dynamics. Journal of Computational Physics 77 (77), 240266.

Dritschel, D. G. 1995 A general theory for two-dimensional vortex interactions. Journal of Fluid Mechanics 293, 269.

Dritschel, D. G. \& WAugh, D. W. 1992 Quantification of the inelastic interaction of unequal vortices in 2-dimensional vortex dynamics. Physics of Fluids A-fluid Dynamics 4 (8), $1737-1744$.

E, W. \& Liu, J.-G. 1996a Finite difference schemes for incompressible flows in vorticity formulations. ESIAM: Proceedings 1, 181-195.

E, W. \& LiU, J.-G. 1996b Vorticity boundary conditions and related issues for finite difference schemes. J. Comp. Phys. 124, 368-382.

Elsas, J. H. \& Moriconi, L. 2017 Vortex identification from local properties of the vorticity field. Physics of Fluids 29 (1), 015101.

Folz, P. J.R. \& Nomura, K. K. 2017 A quantitative assessment of viscous asymmetric vortex pair interactions. Journal of Fluid Mechanics 829, 1-30.

Gallay, T. 2011 Interaction of vortices in weakly viscous planar flows. Archive for Rational Mechanics and Analysis 200 (2), 445-490.

Gallay, T. \& Wayne, C. E. 2005 Global stability of vortex solutions of the two-dimensional navier-stokes equation. Communications in Mathematical Physics 255 (1), 97-129.

Hansen, J. S. 2011 GNU Octave - Beginner's Guide. London: Packt publishing.

Hunt, J. C. R, Wray, A. A. \& Moin, P. 1988 Eddies, streams, and convergence zones in turbulent flows. Tech. Rep. CTR-S88. Center for Turbulence Research, Stanford University.

Jalali, M. M. \& Dritschel, D. G. 2018 The interaction of two asymmetric quasi-geostrophic vortex patches. Geophysical and Astrophysical Fluid Dynamics 112 (6), 375-401.

JAlali, M. M. \& Dritschel, D. G. 2020 Stability and evolution of two opposite-signed quasigeostrophic shallow-water vortex patches. Geophysical and Astrophysical Fluid Dynamics $114(4-5), 561-587$.

Jing, F., Kanso, E. \& Newton, P. K. 2010 Viscous evolution of point vortex equilibria: The collinear state. Physics of Fluids 22 (12), 123102.

Jing, F., Kanso, E. \& Newton, P. K. 2012 Insights into symmetric and asymmetric vortex mergers using the core growth model. Physics of Fluids 24 (7), 073101.

KIM, S. C. \& Sohn, S. I. 2012 Interactions of three viscous point vortices. Journal of Physics A: Mathematical and Theoretical 45 (45), 455501. 
Leweke, T., Le Dizès, S. \& Williamson, C. H.K. 2016 Dynamics and instabilities of vortex pairs. Annual Review of Fluid Mechanics 48 (1), 507-541.

Melander, M. V., Zabusky, N. J. \& McWilliams, J. C. 1988 Symmetric vortex merger in two dimensions: causes and conditions. Journal of Fluid Mechanics 195, 303-340.

Meunier, P., Ehrenstein, U., Leweke, T. \& Rossi, M. 2002 A merging criterion for twodimensional co-rotating vortices. Physics of Fluids 14 (8), 2757-2766.

Nielsen, A. R., Heil, M., Andersen, M. \& Brøns, M. 2019 Bifurcation theory for vortices with application to boundary layer eruption. Journal of Fluid Mechanics 865, 831-849.

Overman, E. A. \& Zabusky, N. J. 1982 Evolution and merger of isolated vortex structures. Physics of Fluids 25 (8), 1297-1305, 1297-1305.

Rutter, J.W. 2000 Geometry of Curves. Taylor \& Francis.

Saffman, P.G. 1992 Vortex dynamics. Cambridge University Press,.

Trieling, R. R., Velasco Fuentes, O. U. \& van Heijst, G. J.F. 2005 Interaction of two unequal corotating vortices. Physics of Fluids 17 (8), 087103.

Zhang, Y., Liu, K., Xian, H. \& Du, X. 2018 A review of methods for vortex identification in hydroturbines. Renewable and Sustainable Energy Reviews 81, 1269-1285. 